\title{
Partial Oxidation-Induced Electrical Conductivity and Paramagnetism in a Ni(II) Tetraaza[14]annulene-Linked Metal Organic Framework
}

Yi Jiang, ${ }^{1, ~ \# ~ I n s e o n ~ O h, ~}{ }^{3,}$ \# Se Hun Joo, ${ }^{4,}$ \# Onur Buyukcakir, ${ }^{1}$ Xiong Chen, ${ }^{1}$ Sun Hwa

Lee, ${ }^{1}$ Ming Huang, ${ }^{1}$ Won Kyung Seong, ${ }^{1}$ Sang Kyu Kwak, ${ }^{4 *}$ Jung-Woo Yoo, ${ }^{3 *}$ and Rodney S. Ruoff ${ }^{1,2,3,4^{*}}$

${ }^{1}$ Center for Multidimensional Carbon Materials (CMCM), Institute for Basic Science (IBS), Ulsan 44919, Republic of Korea

${ }^{2}$ Department of Chemistry Ulsan National Institute of Science and Technology (UNIST), Ulsan 44919, Republic of Korea

${ }^{3}$ School of Materials Science and Engineering, Ulsan National Institute of Science and Technology (UNIST), Ulsan 44919, Republic of Korea

${ }^{4}$ School of Energy and Chemical Engineering, Ulsan National Institute of Science and Technology (UNIST), Ulsan 44919, Republic of Korea

\#These authors contributed equally to this work.

*Email: correspondence and requests for materials should be addressed to R.S.R.

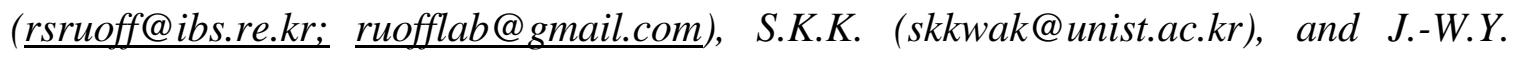
(jwyoo@unist.ac.kr). 


\section{Synthesis of the model compound Ni(II) tetraaza[14]annulene (NiTAA)}

1,2-Phenylenediamine dihydrochloride $(360 \mathrm{mg}, 2 \mathrm{mmol})$ and nickel acetate tetrahydrate (248.8 mg, $1 \mathrm{mmol}$ ) were placed in a reaction flask flushed with Ar, to which water (30 $\mathrm{mL}$ ) was added through a syringe. The mixture was stirred at room temperature under Ar. $\mathrm{Et}_{3} \mathrm{~N}(0.55 \mathrm{~mL}, 4 \mathrm{mmol})$ and 1,1,3,3-tetramethoxypropane $(329 \mu \mathrm{L}, 4 \mathrm{mmol})$ were added sequentially to the above mixture. The mixture was heated at $100{ }^{\circ} \mathrm{C}$ for 24 hours. The precipitated solid was collected by filtration and washed with water ( 3 x $20 \mathrm{~mL})$ and a small amount of methanol $(\sim 5 \mathrm{~mL})$ to yield NiTAA $(268 \mathrm{mg}, 78 \%) .{ }^{1} \mathrm{H}$ NMR $(400 \mathrm{MHz}$, $\left.\mathrm{CD}_{2} \mathrm{Cl}_{2}\right): \delta(\mathrm{ppm}) 7.75(\mathrm{~d}, J=6.4 \mathrm{~Hz}, 4 \mathrm{H}), 7.38(\mathrm{q}, J=3.2 \mathrm{~Hz}, 4 \mathrm{H}), 6.93(\mathrm{q}, J=3.2 \mathrm{~Hz}$, 4H), $5.48(\mathrm{t}, J=6.4 \mathrm{~Hz}, 2 \mathrm{H})$. ESI-MS calculated $m / z=344.1$ for $\left[\mathrm{M}^{+}\right]$, measured $\mathrm{m} / z=$ 344.4. Elemental Anal. Calculated for $\mathrm{C}_{18} \mathrm{H}_{14} \mathrm{~N}_{4} \mathrm{Ni}$ : C, 62.66; H, 4.09; N, 16.24; Found: $\mathrm{C}$, $62.39 ; \mathrm{H}, 4.25 ; \mathrm{N}, 16.02$. 
2. ${ }^{1}$ H NMR spectrum of the model compound NiTAA

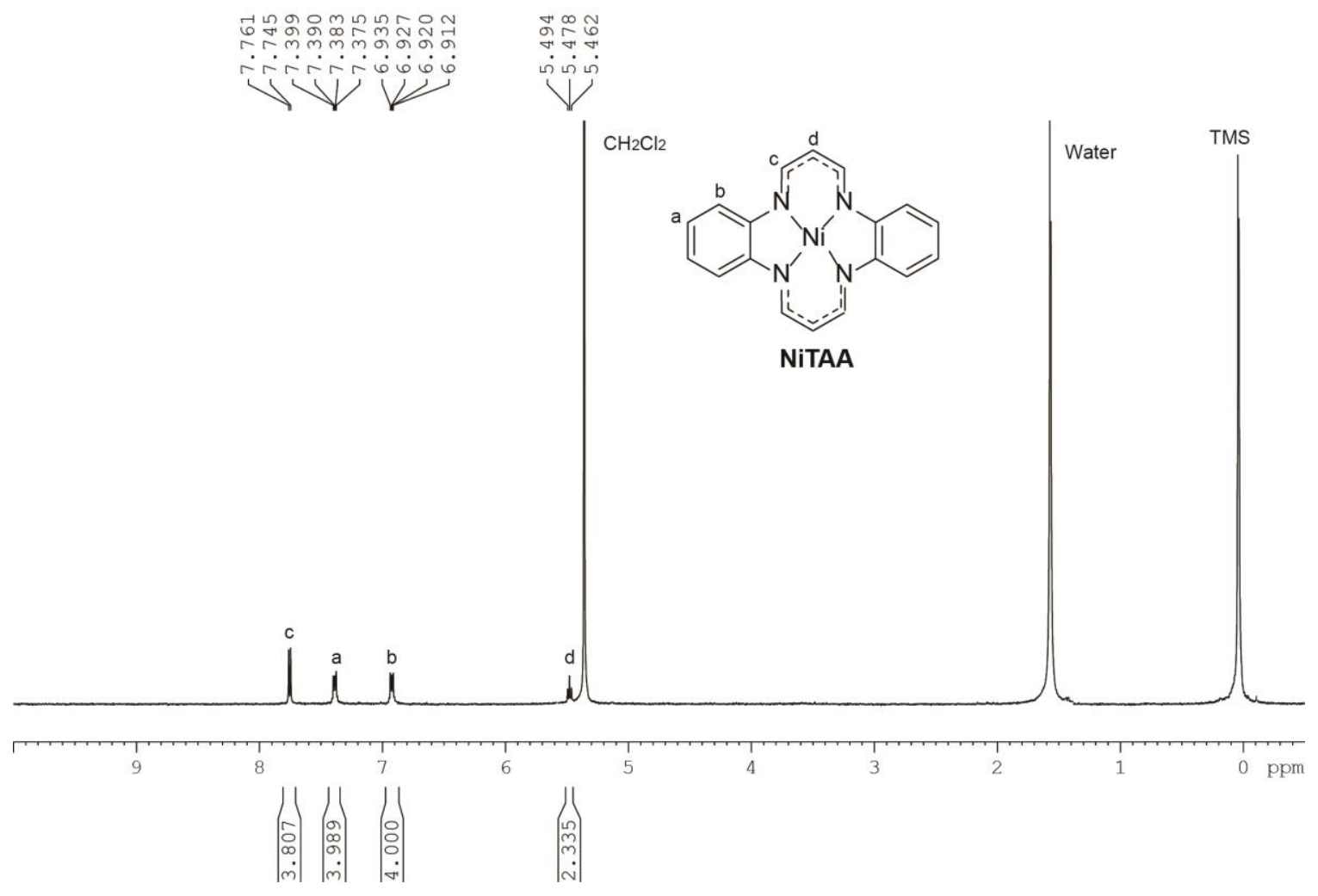

Figure S1. ${ }^{1} \mathrm{H}$ NMR spectrum of the model compound NiTAA $\left(400 \mathrm{MHz}, \mathrm{CD}_{2} \mathrm{Cl}_{2}\right)$. 


\section{Survey XPS spectrum of NiTAA}

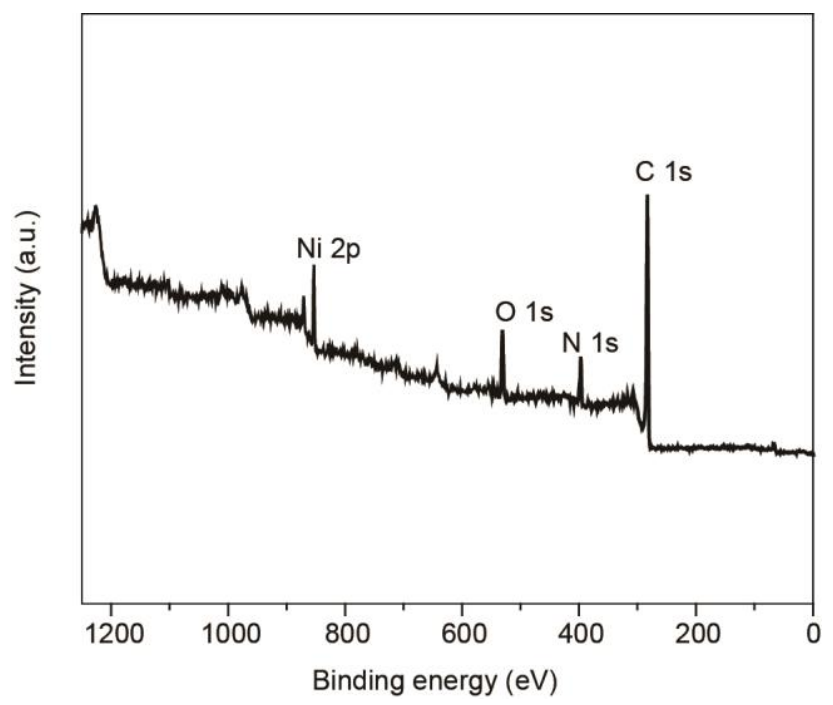

Figure S2. XPS survey spectrum of the model compound NiTAA; no $\mathrm{Cl}^{-}$is detected. 
4. Mechanism of the template $\left(\mathrm{Ni}^{2+}\right)$ synthesis of NiTAA-MOF and the origin of missing-linker defects in NiTAA-MOF

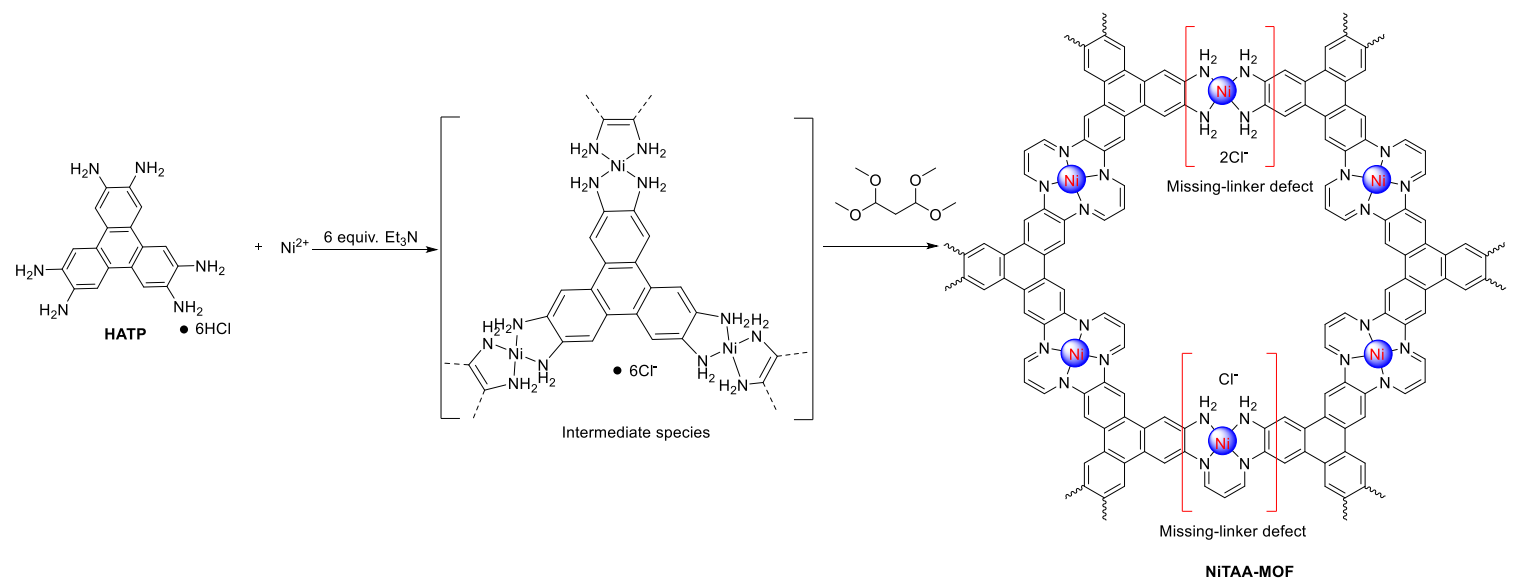

Scheme S1. Mechanism of the template synthesis of NiTAA-MOF and the origin of missing-linker defects in NiTAA-MOF. Missing-linker defects are attributed to the presence of unreacted intermediate species during template synthesis. 
5. Diffuse reflectance UV-vis spectrum of NiTAA-MOF.

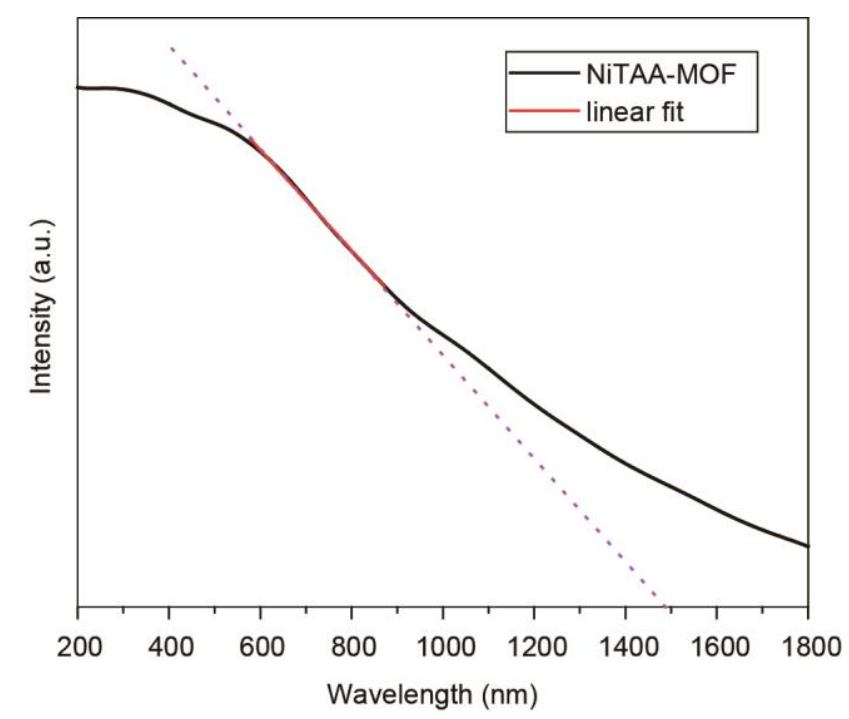

Figure S3. Diffuse reflectance UV-vis spectrum of NiTAA-MOF from which a band-gap of $0.83 \mathrm{eV}(1487 \mathrm{~nm})$ was determined by extrapolation. 


\section{Density functional theory calculations}

Spin-polarized density functional theory (DFT) calculations were performed using Vienna ab initio simulation package (VASP). ${ }^{1,2}$ The generalized gradient approximation with the Perdew-Burke-Ernzerhof functional (GGA-PBE) was used to describe the exchangecorrelation potential of the electrons. ${ }^{3}$ The electron-ion interaction was described by the projector-augmented-wave (PAW) method. ${ }^{4}$ The long-range van der Waals interactions were corrected using the DFT-D3 method by Grimme. ${ }^{5}$ The Hubbard $U$ correction was applied to the $\mathrm{Ni} 3 d$ electrons $\left(U_{\mathrm{eff}}=6.4 \mathrm{eV}\right.$ for the $d$ electrons of $\left.\mathrm{Ni}\right){ }^{6,7}$ The wave functions were expanded using a plane-wave basis set with a kinetic energy cutoff of $600 \mathrm{eV}$. The Brillouin zone was sampled by a $1 \times 1 \times 3$ k-point set with the Monkhorst-Pack scheme. ${ }^{8}$ The self-consistent field calculation was performed with the convergence criterion of 1.0 $\times 10^{-6} \mathrm{eV}$. Geometry optimization was carried out, until the Hellmann-Feynman forces acting on ions were less than $0.01 \mathrm{eV} / \AA$. The potential energy surface was generated using two different grids (i.e., $9 \times 9$ and $7 \times 7$ grids) of $a b$-plane displacements for sampling structures, each consisting of 81 and 49 different unit cells, respectively. For each grid, the potential energy surface was constructed by interpolating the total energies calculated by DFT over a $1000 \times 1000$ fine grid using the bicubic spline method. In the band gap calculation, the GGA-PBE functional usually underestimates the electronic band gap value. To provide a more accurate theoretical electronic band gap of the NiTAA-MOF, we also used the HSE06 hybrid functional for the band gap calculation. ${ }^{9,} 10$ The band gap value calculated with the HSE06 hybrid functional was found to be $1.70 \mathrm{eV}$. 
7. The DFT total energies of NiTAA-MOF with slipped-parallel I and II stacking modes.

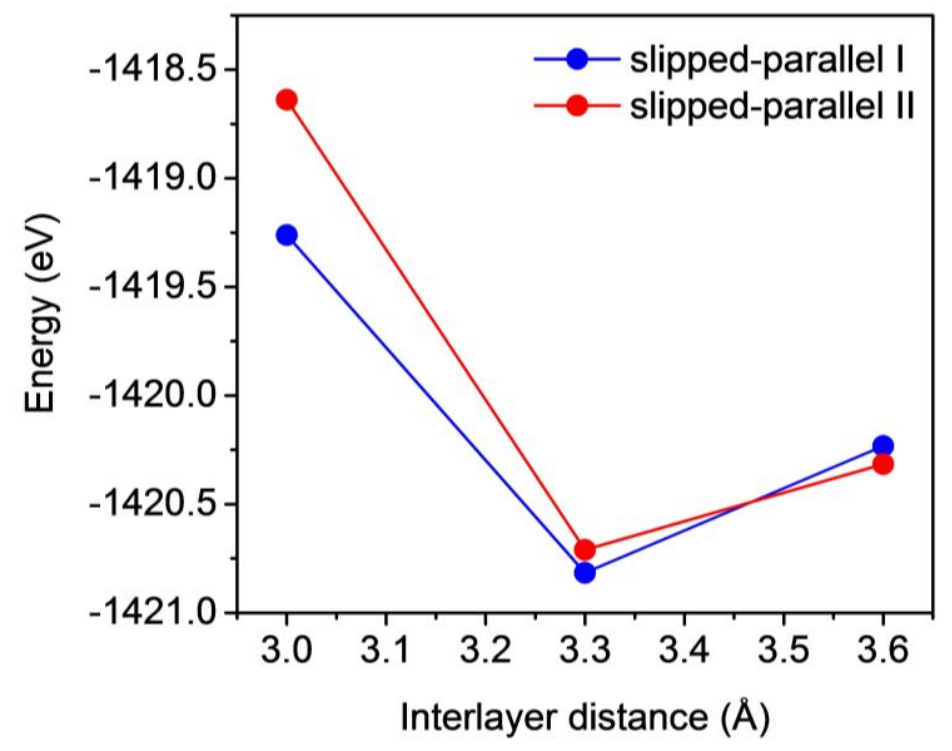

Figure S4. The DFT total energies of NiTAA-MOF with slipped-parallel I and II stacking modes at interlayer distances of $3.0 \AA$ A, $3.3 \AA$, and $3.6 \AA$. 
8. HR-TEM image of the as-synthesized NiTAA-MOF.

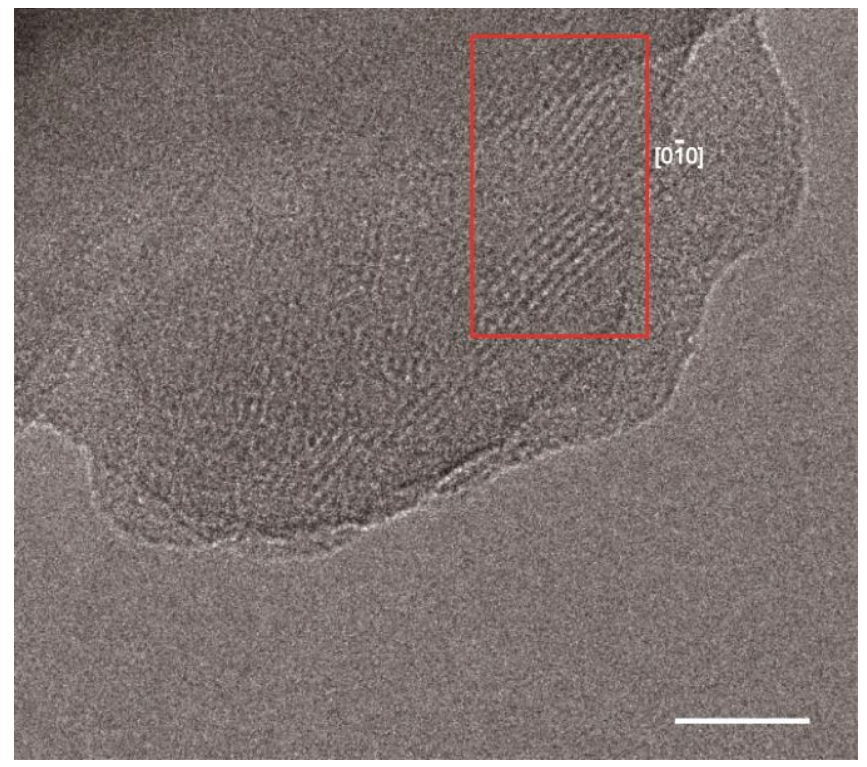

Figure S5. HR-TEM image of the as-synthesized NiTAA-MOF. Scale bar: $20 \mathrm{~nm}$. An operating voltage of $200 \mathrm{kV}$ was used for HR-TEM imaging. 
9. HR-TEM image of the partially oxidized NiTAA-MOF.

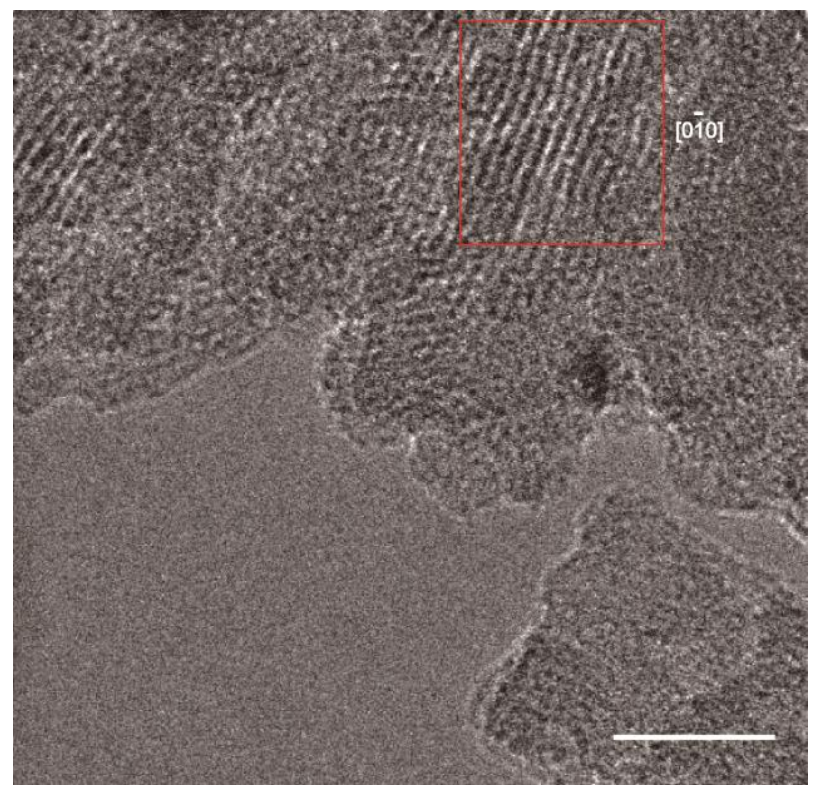

Figure S6. HR-TEM image of the partially oxidized NiTAA-MOF. Scale bar: $20 \mathrm{~nm}$. An operating voltage of $200 \mathrm{kV}$ was used for HR-TEM imaging. 


\section{Nitrogen sorption analysis.}
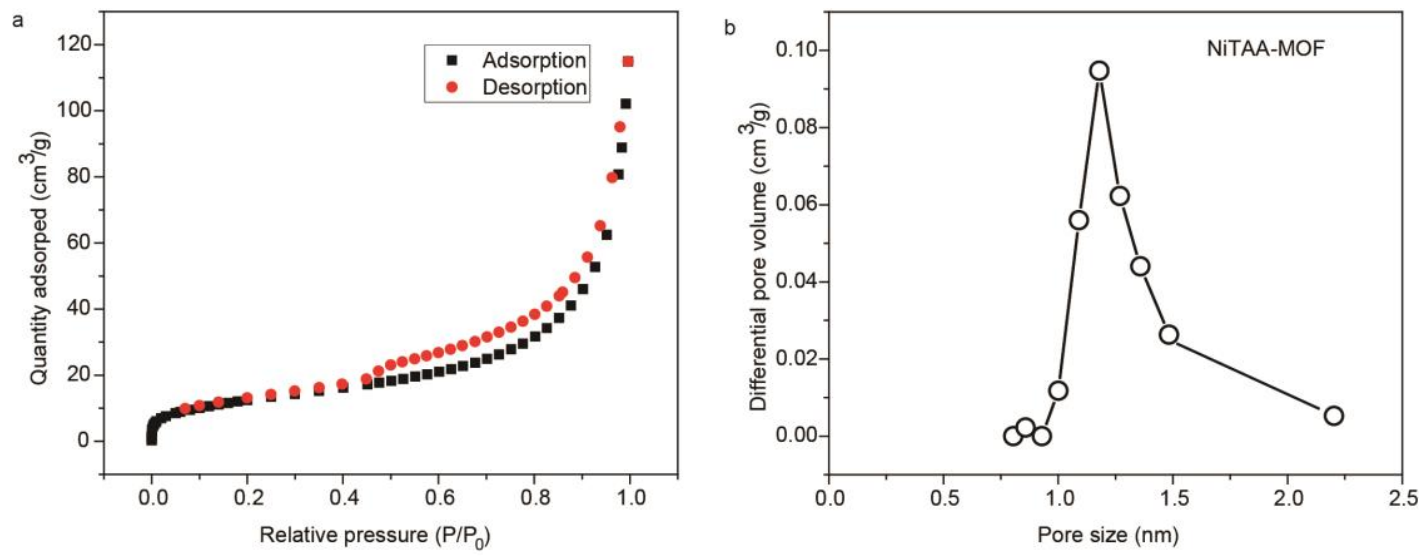

Figure S7. $\mathrm{N}_{2}$ sorption analysis of NiTAA-MOF. (a) $\mathrm{N}_{2}$ sorption isotherms of NiTAAMOF powder. (b) Pore size distribution of NiTAA-MOF powder. 


\section{High-resolution C1s XPS spectrum of NiTAA-MOF.}

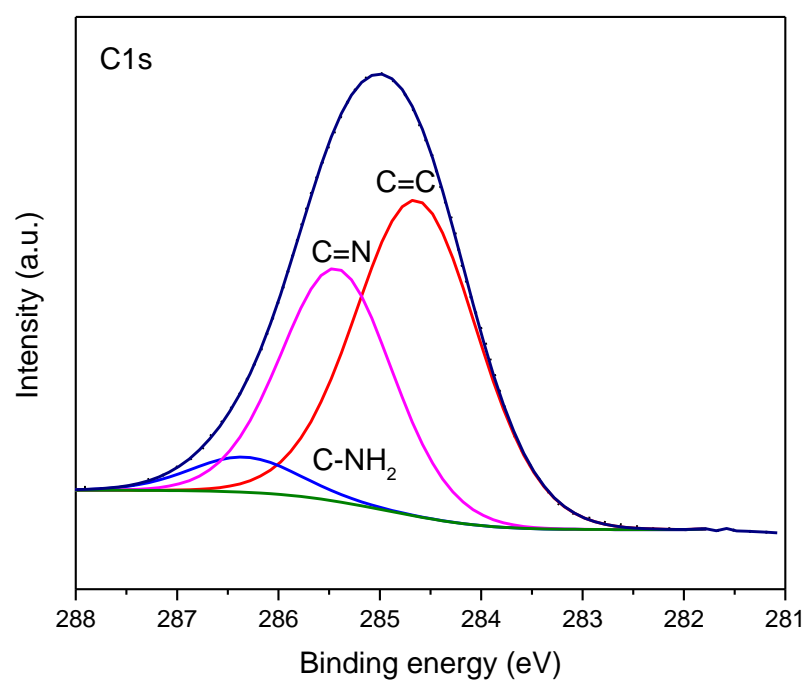

Figure S8. High-resolution C1s XPS spectrum and the corresponding curve fitting results of NiTAA-MOF. 
12. Thermo gravimetric analysis (TGA) of NiTAA-MOF.

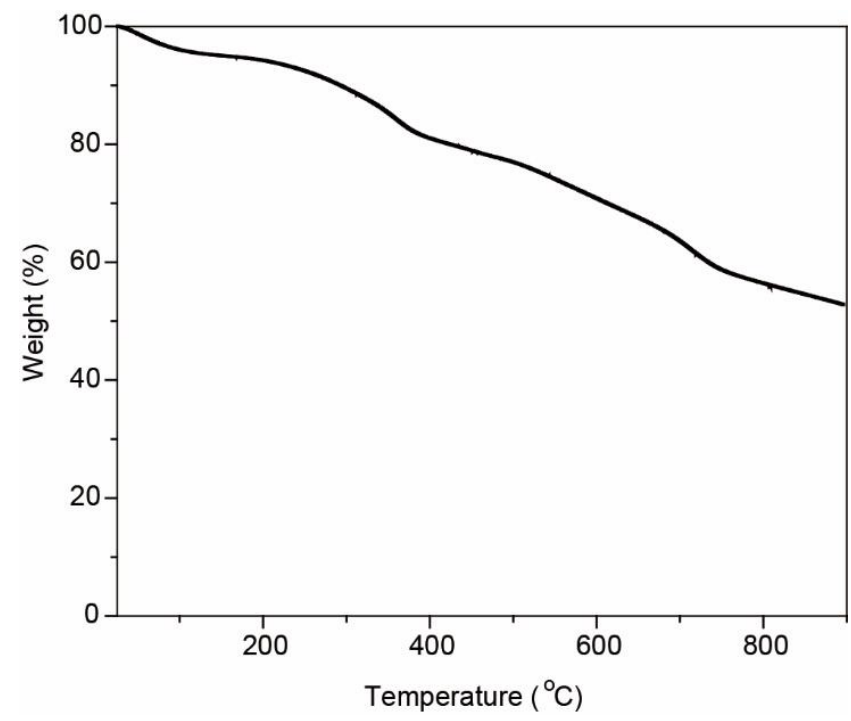

Figure S9. TGA of NiTAA-MOF under nitrogen by heating to $900{ }^{\circ} \mathrm{C}$ at the rate of $5{ }^{\circ} \mathrm{C}$ $\min ^{-1}$ 
13. Elemental analysis of NiTAA-MOF

\begin{tabular}{|c|c|c|c|}
\hline & C(wt. \%) & H (wt. \%) & N (wt. \%) \\
\hline NiTAA-MOF & 55.69 & 3.52 & 15.86 \\
\hline$\left(\mathrm{C}_{48} \mathrm{H}_{32} \mathrm{~N}_{12} \mathrm{Ni}_{3} \mathrm{Cl}_{2}\right)_{\mathrm{n}}$ & 56.31 & 3.15 & 16.42 \\
$($ Calculated $)$ & & & \\
\hline
\end{tabular}

Table 1. Elemental analysis data for NiTAA-MOF, based on which the chemical formula of NiTAA-MOF can be written as $\left(\mathrm{C}_{48} \mathrm{H}_{32} \mathrm{~N}_{12} \mathrm{Ni}_{3} \mathrm{Cl}_{2}\right)_{n}$ revealing the presence of missing-linker defects, also deduced from XPS of NiTAA-MOF. 
14. Concentrations of selected metals $(\mathrm{mg} / \mathrm{Kg})$ in NiTAA-MOF as determined by ICP-MS.

\begin{tabular}{|c|c|c|c|c|c|c|c|}
\hline $\mathrm{Ni}$ & $\mathrm{Co}$ & $\mathrm{Li}$ & $\mathrm{K}$ & $\mathrm{Mn}$ & $\mathrm{Na}$ & $\mathrm{Fe}$ & $\mathrm{Cd}$ \\
\hline 151898 & 8.65 & - & - & 0.36 & 153 & 15.6 & 1.01 \\
\hline $\mathrm{Zn}$ & $\mathrm{Cu}$ & $\mathrm{Mg}$ & $\mathrm{Al}$ & & & & \\
\hline 20.5 & 5.97 & - & 16.3 & & & & \\
\hline
\end{tabular}




\section{Chemical stability of NiTAA-MOF}

a

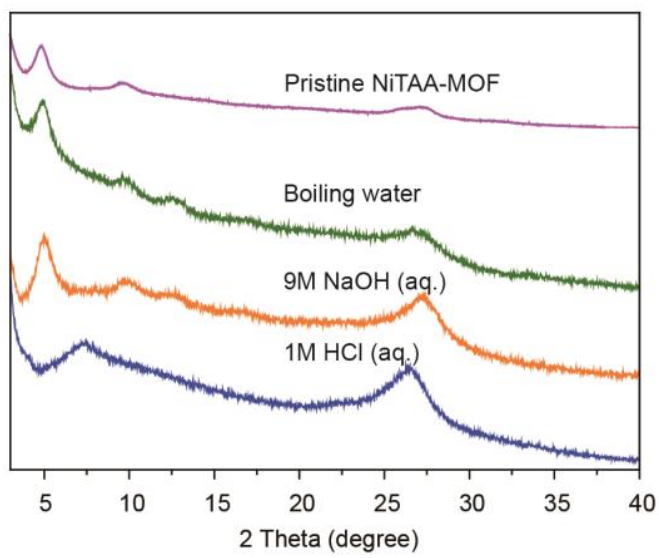

b

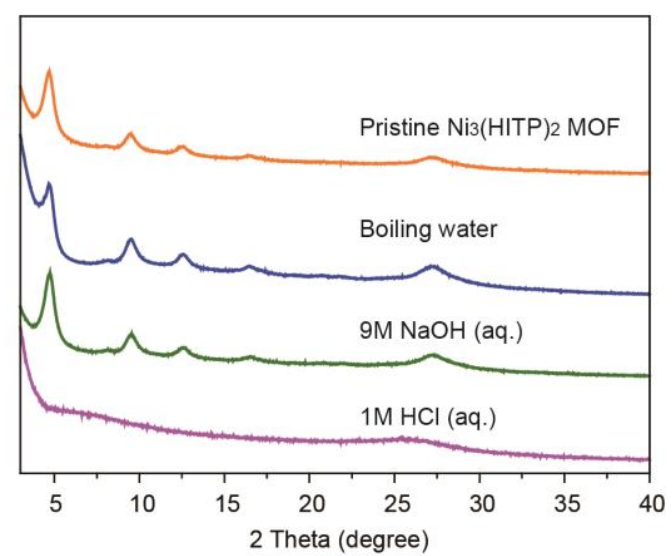

Figure S10. XRD patterns of NiTAA-MOF (a) and $\mathbf{N i}_{3}(\mathbf{H I T P})_{2} \mathrm{MOF}^{11}$ (b) after treatment for 7 days in boiling water and $9 \mathrm{M} \mathrm{NaOH}$ (aq.), and 1 day in $1 \mathrm{M} \mathrm{HCl}$ (aq.). 
16. PXRD pattern of iodine-doped NiTAA-MOF

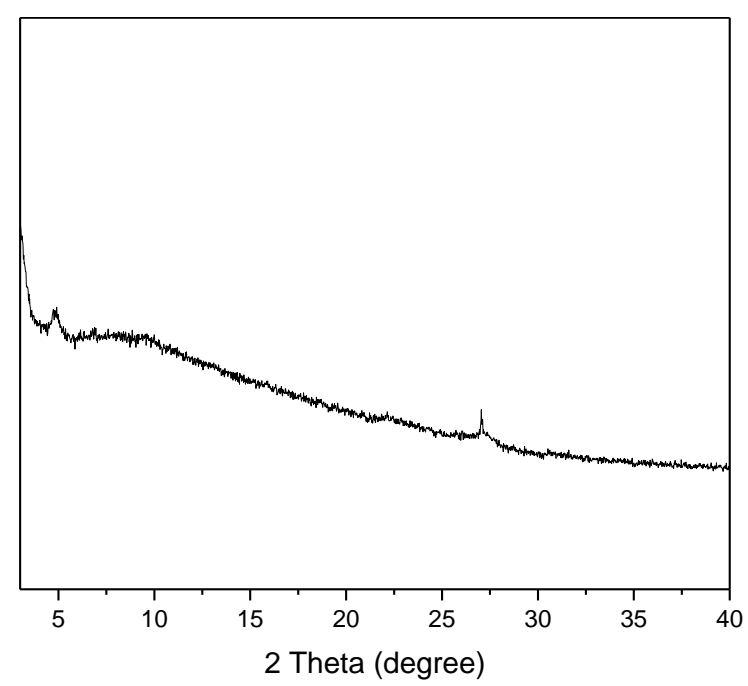

Figure S11. XRD pattern of the partial oxidized NiTAA-MOF. 
17. Concentrations of selected metals $(\mathrm{mg} / \mathrm{Kg})$ in iodine-doped NiTAA-MOF determined by ICP-MS.

\begin{tabular}{|c|c|c|c|c|c|c|c|}
\hline $\mathrm{Ni}$ & $\mathrm{Co}$ & $\mathrm{Li}$ & $\mathrm{K}$ & $\mathrm{Mn}$ & $\mathrm{Na}$ & $\mathrm{Fe}$ & $\mathrm{Cd}$ \\
\hline 112065 & 11.8 & 1.06 & 56.4 & 1.39 & 89.2 & 8.76 & 0.21 \\
\hline $\mathrm{Zn}$ & $\mathrm{Cu}$ & $\mathrm{Mg}$ & $\mathrm{Al}$ & & & & \\
\hline 25.9 & 4.07 & - & 1.57 & & & & \\
\hline
\end{tabular}




\section{References}

1. Kresse, G.; Furthmüller, J. Efficient Iterative Schemes for Ab Initio Total-Energy Calculations Using a Plane-Wave Basis Set. Phys. Rev. B 1996, 54, 11169-11186.

2. Kresse, G.; Furthmüller, J. Efficiency of Ab-Initio Total Energy Calculations for Metals and Semiconductors Using a Plane-Wave Basis Set. Comp. Mater. Sci. 1996, 6, 15-50.

3. Perdew, J. P.; Burke, K.; Ernzerhof, M. Generalized Gradient Approximation Made Simple. Phys. Rev. Lett. 1996, 77, 3865-3868.

4. Kresse, G.; Joubert, D. From Ultrasoft Pseudopotentials to the Projector AugmentedWave Method. Phys. Rev. B 1999, 59, 1758-1775.

5. Grimme, S.; Antony, J.; Ehrlich, S.; Krieg, H. A Consistent and Accurate Ab Initio Parametrization of Density Functional Dispersion Correction (DFT-D) for the 94 Elements H-Pu. J. Chem. Phys. 2010, 132, 154104.

6. Liechtenstein, A. I.; Anisimov, V. I.; Zaanen, J. Density-Functional Theory and Strong Interactions: Orbital Ordering in Mott-Hubbard Insulators. Phys. Rev. B 1995, 52, R5467.

7. Wang, L.; Maxisch, T.; Ceder, G. Oxidation Energies of Transition Metal Oxides within the GGA + U Framework. Phys. Rev. B 2006, 73, 195107.

8. Monkhorst, H. J.; Pack, J. D. Special Points for Brillouin-Zone Integrations. Phys. Rev. B 1976, 13, 5188-5192.

9. Heyd, J.; Scuseria, G. E.; Ernzerhof, M. Hybrid Functionals Based on a Screened Coulomb Potential. J. Chem. Phys. 2003, 118, 8207-8215. 
10. Krukau, A. V.; Vydrov, O. A.; Izmaylov, A. F.; Scuseria, G. E. Influence of the Exchange Screening Parameter on the Performance of Screened Hybrid Functionals. $J$. Chem. Phys. 2006, 125, 224106.

11. Sheberla, D.; Sun, L.; Blood-Forsythe, M. A.; Er, S.; Wade, C. R.; Brozek, C. K.; Aspuru-Guzik, A.; Dincă, M. High Electrical Conductivity in Ni $\mathrm{Ni}_{3}(2,3,6,7,10,11$ hexaiminotriphenylene)2, a Semiconducting Metal-Organic Graphene Analogue. J. Am. Chem. Soc. 2014, 136, 8859-8862. 